\title{
Transfusion Medicine
}

Infusionstherapie

Transfusionsmedizin

15 Einige Gedanken Ihres Verlegers

Karger, T.; Karger, S. (Basel)

Originalarbeiten

16 Einführung eines durchflusszytometrischen Typisierungsverfahrens zur schnellen Erkennung HPA-1-kompatibler Thrombozytenspender unter Routinebedingungen

Ostendorf, N.; Stahl, D.; Sibrowski, W. (Münster)

112 Vergleich von Thrombozytenkonzentraten zweier Thrombozytapherese-Programme der $\mathrm{MCS}^{{ }^{\circledR}}+$ Stiegler, G.; Leitner, G.; Panzer, S.; Höcker, P. (Wien)

118 Fünf-Tage-Lagerung von Apherese-Thrombozytenkonzentraten in der neuen Additivlösung T-Sol ${ }^{\mathrm{TM}}$ : Veränderungen der metabolischen Parameter Stiegler, G.; Leitner, G.; Jurko, S.; Gerhartl, K.; Panzer, S.; Höcker, P. (Wien)

257 Zeitabhängige Veränderungen der Funktion von Steroid- versus G-CSF-mobilisierten Ganulozyten: Überlegungen zur Lagerung von Ganulozytenkonzentraten für Patienten mit Neutropenie Schmitt, A.; Reinhardt, P.; Schmitt, M.; Nowak-Hanau, S.; Maccari, B.; Schulz, A.; Kubanek, B.; Wiesneth, M. (Ulm)

4193 Der Einfluss der photochemischen Behandlung mit Amotosalenhydrochlorid und UV-A-Licht auf die Qualität von Apherese-Thrombozytenkonzentraten Janetzko, K. (Mannheim); Klinger, M. (Lübeck); Mayaudon, V. (Nivelles); Lin, L. (Concord, CA); Eichler, H.; Klüter, H. (Mannheim)

4200 Häufigkeit von Nebenwirkungen während Blut- und Apheresespenden: Eine Single-Center-Studie Franchini, M; Gandini, G.; Gandini, A.R.; Crocco, I.; de Gironcoli, M.; Bertuzzo, D.; Guiffrida, A.C.; Lippi, G.; Vassanelli, A.; Bressan, F.; Aprili, G. (Verona)

5249 Diagnostische Bedeutung und Schwierigkeiten bei der Bestimmung der T-Antigen-Freilegung Strobel, E. (München)

5254 Entwicklung und Validierung eines NAT-Verfahrens zum Screenen von Blutspenden auf Parvovirus B19 DNA

Müller, J.; Eis-Hübinger, A.M. (Bonn); Madlener, K. (Bad Nauheim); Küppers, C.; Herzig, M.; Pötzsch, B. (Bonn)
5259 Untersuchungen zur Qualität von Erythrozytenkonzentraten nach mechanisiertem Transport in einem modernen Rohrpostsystem Hellkamp, J.; Carl, A.; Kohse, K.P. (Oldenburg)

5265 Eine rationale medizinische Entscheidungsfindung verbessert die Wirksamkeit und Kosteneffizienz der autologen Bluttransfusion: Präoperative Blutspende, perioperative maschinelle Autotransfusion und präoperative autologe Plasmapherese Singbartl, G. (Hamburg); Schleinzer, W. (Nottwil); Munkel, H. (Hamburg)

6305 Infektionen mit HIV, HBV und HCV unter Blutspendern in Deutschland 1998 und 1999 Stark, K.; Werner, E.; Seeger, E.; Offergeld, R.; Altmann, D.; Kramer, M.H. (Berlin)

6308 Kontinuierliche Autotransfusion bei Zeugen Jehovas nach kardiopulmonalem Bypass Schmidt, C.; Van Aken, H.; Asfour, B.; Stanojevic, D.; Booke, M. (Münster)

6313 Das Auftauen von Gefrierplasma und virusinaktiviertem Plasma - die Eignung von Mikrowellengeräten Beck, K.H.; Schüftan, C.; Schubert, D.; Kretschmer, V. (Marburg)

\section{Übersichtsarbeiten}

125 Kryokonservierung von Erythrozyten durch Einfrieren und Vitrifizieren - Erinnerungen und Voraussagen Rowe, A.W. (New York)

267 Lokale Anwendung von Thrombozytenkonzentraten zur Geweberegeneration Dugrillon, A.; Klüter, H. (Mannheim)

4206 Serologische und klinische Aspekte von autoimmunhämolytischen Anämien Salama, A.; Ahrens, N.; Kiesewetter, H. (Berlin)

4218 Ökonomie der Transfusion Yeh, J.M.; Botteman, M.; Pashos, C.L. (Cambridge, MA); Postma, M.J. (Groningen); Staginnus, U. (Madrid)

5271 Die transfusionsassoziierte akute Lungeninsuffizienz: Eine wenig bekannte jedoch lebensbedrohliche Transfusionsreaktion Bux, J. (Gießen)

\begin{tabular}{ll}
\hline KARGER & ( 2002 S. Karger GmbH, Freiburg \\
Fax +497614520714 & Accessible online at: \\
$\begin{array}{l}\text { E-mail Information@Karger.de } \\
\text { www.karger.com }\end{array}$ & www.karger.com/journals/iut
\end{tabular}


6318 Die Relevanz von Blutgruppenunterschieden und Antikörpern bei der allogenen Stammzelltransplantation Cassens, U.; Sibrowski, W. (Münster)

6326 Assoziation bestimmter HLA-Marker mit, der Schuppenflechte - Revision

Eiermann, T.H. (Hamburg); Vejbaesya, S. (Bangkok); Prestel, H.; Roepke, A.; Müller-Myhsok, B. (Hamburg); Schmitt-Egenolf, M. (Umea)

Kommentare

131 Qualitätsverbesserung durch Gesetze und Richtlinien. Anmerkungen zur behördlichen Überwachung bei der Implementierung qualitätssichernder Maßnahmen in der Hämotherapie

Heim, M.U. (Magdeburg)

4232 Plasma zur Fraktionierung: Wirkstoff oder

Arzneimittel?

Hasskarl, H.; Ziegler, U. (Ludwigshafen)

6338 Der externe Transfusionsverantwortliche. Zu Hasskarl H: Transfusiosngesetz (TFG) vom 1. Juli 1998 (BGBl. I S. 1752): Bestellung eines nicht institutionsangehörigen Transfusionsverantwortlichen - gutachterliche Stellungnahme. Infus Ther Transfus Med 2002;29:331-337

Krausbauer, U. (Koblenz); Kretschmer, V. (Marburg)

6341 Antwort des Autors

Hasskarl, H. (Ludwigshafen)

\section{Klinische Information}

6331 Transfusionsgesetz vom 1. Juli 1998 (BGBI. I S. 1752): Bestellung eines nicht institutsangehörigen Transfusionsverantwortlichen - gutachterliche Stellungnahme Hasskarl, H. (Ludwigshafen)

Kasuistik

5277 Ergebnisse der Behandlung von 3 Patienten mit idiopathischer autoimmunhämolytischer Anämie und/oder Immunthrombozytopenie mit Rituximab und kurze Übersicht über die publizierten Fälle

Ahrens, N.; Heymann, G.; Meyer, O.; Kiesewetter, H.; Salama, A. (Berlin)

Qualitätsmanagement

273 Blut und Blutkomponenten - Aktuelle Arbeit des Council of Europe

Bopp, K.-F. (Straßburg)
4226 Eurocode-Blutkennzeichnungssystem - die einheitliche Identifikation und Klasssifikation von Blutprodukten erhöht die Transfusionssicherheit

Knels, R. (Dresden); Roos, D. (Hamburg); Boecker, W. (Hagen); Kardoeus, J. (Münster); Becker, G. (München)

Briefe an die Herausgeber

135 Ausschluss von Spendern nach Transplantation von Gewebe nichthumanen Ursprungs in Deutschland Caspari, G. (Greifswald); Gerlich, W.H. (Gießen); Gürtler, L. (Greifswald)

5282 Zu Innerhofer P, Kühlbacher G: Immunomodulation mechanisms following transfusion of allogeneic and autologous erythrocyte concentrates.

Infus Ther Transfus Med 2002;29:118-121.

Leo, A. (Heidelberg)

6343 Stellungnahme zur Heranziehung von Personen mit Hämatochromatose zur Blutspende Kretschmer, V. (Marburg)

\section{Erratum}

Abstracts

277 9. Jahrestagung der Deutschen Gesellschaft für Immungenetik

Gast-Herausgeber: Seidl, C. (Frankfurt/M.); Holzberger, G. (Kassel)

5283 10. Jahrestagung der Deutschen Gesellschaft für Immungenetik

Gast-Herausgeber: Kappelitz, D.; Neppert, J (Kiel)

Buchbesprechungen

137, 4237

Gesellschaftsmitteilungen

1 38, 2 97, 6344

Industrieforum

1 45, 3 184, 4 238, 5296

Tagungen und Kongresse

1 47, 2 98, 3 186, 4 241, 5 297, 6345 
Themenheft

Autologous Transfusion - from Euphoria to Reason:

Clinical Practice Based on Scientific Knowledge.

CAT Concept Autologe Transfusion - 2nd International

Symposium, Nottwil, January 18-19, 2002

Gast-Herausgeber: Singbartl, G. (Hamburg); Schleinzer, W. (Nottwil)

3107 Autologe Transfusion - von der Euphorie zur Ratio: Praktisches Handeln aus wissenschaftlicher Erkenntnis Singbartl, G. (Hamburg); Schleinzer, W. (Nottwil)

3110 Autologe Transfusion - Pro und Contra aus gesundheitspolitischer Sicht

Von Auer, F. (Bonn)

3118 Immunmodulationsmechanismen nach Transfusion von allogenen und autologen Erythrozytenkonzentraten Innerhofer, P.; Kühlbacher, G. (Innsbruck)

3122 Immunmodulation nach Bluttransfusion - Relevanz für die Inzidenz postoperativer Infektionen Hobisch-Hagen, P. (Innsbruck)

3125 Physikalische Grundlagen der maschinellen

Autotransfusion

Radvan, J. (Aachen); Singbartl, G. (Hamburg); Heschel, I.; Rau, G. (Aachen)

3134 Leukozytenaktvierung mittels maschineller Aotutransfusion

Innerhofer, P.; Wiedermann, F.J. (Innsbruck)

3138 Intraoperative Autotransfusion in der onkologischen Chirurgie - Antworten auf aktuelle Fragen Hansen, E.; Bechmann, V.; Altmeppen, J. (Regensburg)

3142 Maschinelle Autotransfusion bei speziellen Eingriffen und besonderen Begleiterkrankungen Dietrich, G.V. (Eggenfelden)
3147 Vorschlag zum internen Qualitätsmanagement in der intra- und postoperativen Autotransfusion Hansen, E.; Altmeppen, J. (Regensburg)

3151 Aprotinin - eine blutsparende Substanz Haas, S. (München)

3156 Identischer Transfusionstrigger bei autologen und homologen Konserven?

Dietrich, G.V. (Eggenfelden)

3160 Sicherheit von präoperativen autologen Blutspenden in der Herzchirurgie

Walpoth, B.H.; Aregger, F.; Imboden, C.; Auckenthaler, E.; Nydegger, U.; Carrel, T. (Bern)

3163 Perioperative Anämietoleranz Gombotz, H.; Schatz, E. (Linz)

3167 Künstliche Sauerstoffträger - eine aktuelle kritische Analyse

Dinkelmann, S.; Northoff, H. (Tübingen)

3175 Eine gesundheitsökonomische Analyse der autologen Transfusion

Sonnenberg, F.A. (New Brunswick, NJ)

6348 Autorenverzeichnis 2002

6351 Sachwortverzeichnis 2002

Supplement 1

35. Jahreskongress der Deutschen Gesellschaft für Transfusionsmedizin und Immunhämatologie e.V. München, 3.-6. September 2002. Abstracts

Gast-Herausgeber: Mempel, W.; Schramm, W. (München) 